\section{A case of Aggregatibacter actinomycetemcomitans endocarditis presenting as quadriceps myositis}

\author{
Angeline Reid, ${ }^{1}$ Katerina Liew, ${ }^{1}$ \\ Peter Stride, ${ }^{1}$ Robert Horvath, ${ }^{2}$ \\ Jonathan Hunter, ${ }^{1}$ Mostafa Seleem ${ }^{1}$ \\ 'University of Queensland School of \\ Medicine, Redcliffe Hospital, Redcliffe, \\ Queensland; 2Prince Charles Hospital, \\ Brisbane, Queensland, Australia
}

\section{Abstract}

An 80 year old female was admitted with an eight week history of fever associated with painful swelling of her right thigh, and a long history of poor dentition. Culture of blood stained fluid aspirated from the abscess grew Aggregatibacter actinomycetemcomitans (Aa) sensitive to ampicillin and cephalosporins. Transoesophageal echocardiography indicated endocarditis. Four weeks treatment with intravenous ceftriaxone and appropriate dental care was followed by full recovery.

\section{Case Report}

An 80 year old female was admitted with an eight week history of fever associated with painful swelling of her right thigh, currently being treated with flucloxacillin. She had a four year history of hyperlipidaemia treated with simvastatin $40 \mathrm{mg}$ nocte and type II diabetes treated with gliclazide MR (modified release) $30 \mathrm{mg}$ daily. Other current therapy included frusemide, budesonide, alprazolam, aspirin, esomeprazole, sertraline, ramipril and paracetamol. She denied previous cardiac disease, recent travel or contacts with animals. Several months previously she had attended a dentist who noted local infection and advised removal of a tooth, though this was not performed.

On examination temperature was 39.7 , and other observations normal. Dental examination revealed infection of the lower right second molar requiring extraction, plus minor decay of three other teeth and some periodontal disease. She had no peripheral stigmata of endocarditis and normal cardiac sounds with no added murmur. The right thigh was diffusely swollen and tender.

\section{Investigations}

Hb 84 g/L (RR 110-165g/L), WBC 15.9×10

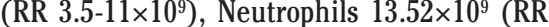
$2-8 \times 10^{9}$ ), CRP $327 \mathrm{mg} / \mathrm{L}(\mathrm{RR}<5 \mathrm{mg} / \mathrm{L})$, glucose $23.3 \mathrm{mmol} / \mathrm{L}$ (RR 3-7.8 mmol/L), HbAlc 9.3\% (4-6\%), Albumen 22g/L (RR 35-50), Alkaline phosphatase 151U/L (RR 53-141)

Blood culture - no growth (Figure 1).

Aspiration from the abscess yielded blood stained fluid. After 2 days of incubation, a small Gram-negative bacillus was cultured. This was identified by Vitek-2 as Aggregatibacter actinomycetemcomitans (Aa) From disc sensitivity tests it was reported sensitive to ampicillin and cephalosporins.

Transoesophageal echocardiography (TOE) included careful scrutiny of the aortic arch detected two small $3 \mathrm{~mm}$ long echodensities on the mitral valve leaflets, not present on repeat TOE after two weeks antibiotic therapy, but no other abnormality. She was treated with ceftriaxone $1 \mathrm{gm}$ twice daily intravenously for four weeks for probable endocarditis with full recovery. Fever abated, pain and swelling resolved and CRP fell to normal.

\section{Discussion}

Aggregatibacter actinomycetemcomitans (previously Actinobacillus actinomycetemcomitans) is a gram-negative coccobacillus, found as an oral commensal, which may also cause severe infections in the oral cavity, particularly periodontitis. It is one of the HACEK group of microorganisms which cause $3 \%$ of all cases of infective endocarditis (IE) ${ }^{1}$ A.actinomycetemcomitans is a virulent microorganism with many protective mechanisms; it produces a leukotoxin which kills neutrophils and monocytes, it inhibits antibody production and activated T-suppressor cells, it is resistant to complement mediated killing, and has immunosuppressive factors that inhibit blastogenesis. The micro-organism is relatively susceptible to antibiotics active against gram negative bacteria. ${ }^{2}$

A.actinomycetemcomitans infection at sites other than oral cavity or cardiac valves is rare. ${ }^{1,2}$ Direct spread from the oral cavity, commonly in the setting of clinical periodontitis, to the jaw, lower respiratory tract, parotid and thyroid glands and brain ${ }^{3}$ are documented. Infection of superficial lesions with oral flora, usually in the setting of trauma, is another common cause of infection with this rare organism. ${ }^{2}$ Widespread peripheral emboli from haematogenous spread in the presence of endocarditis is well documented. ${ }^{1}$

Although non oral infections are rare, bacterial endocarditis is the commonest infection outside the oral cavity. A.actinomycetemcomitans was first described as an etiological agent in endocarditis in 1964 by Mitchell and Gillespie. ${ }^{4}$ Kaplan $^{2}$ described 15 personal
Correspondence: Peter Stride, Redcliffe Hospital, Redcliffe, Queensland 4020 Australia. Fax +61.7.3883.7975

E-mail: peter_stride@health.qld.gov.au

Key words: aggregatibacter actinomycetemcomitans, myositis, HACEK, endocarditis.

Received for publication: 12 October 2011. Revision received: 20 December 2011. Accepted for publication: 8 January 2012.

This work is licensed under a Creative Commons Attribution NonCommercial 3.0 License (CC BYNC 3.0).

(C) Copyright A. Reid et al., 2012

Licensee PAGEPress srl, Italy

Infectious Disease Reports 2012; 4:e14

doi:10.4081/idr.2012.e14

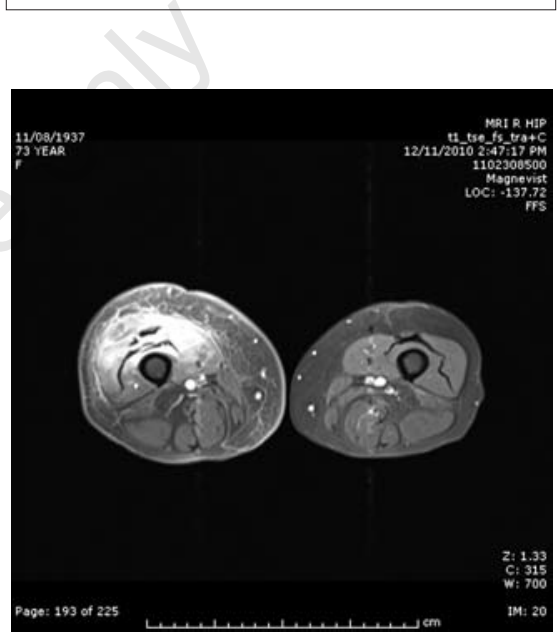

Figure 1. Magnetic resonance imaging scan (T1 fat saturated image) detected diffuse oedema with contrast enhancement involving the right extensor thigh muscles compatible with infection or myositis.

cases of A.actinomycetemcomitans where infection was confined to the head or cardiac valves or lower respiratory tract, and reported that 21 out of 57 documented up to 1989 had poor dentition. Pasture ${ }^{1}{ }^{\text {recorded }} 99$ cases of endocarditis reported up to 2004 , and found $75 \%$ of patients had previous heart disease before infective endocarditis, the portal of entry of which was usually the oral cavity. The aortic valve is most commonly involved.

The onset of endocarditis is usually insidious, with a mean duration of 13 weeks symptoms before diagnosis is confirmed by blood cultures incubated for $>5$ days. Intermittent fever, weight loss, peripheral signs of endocarditis, anaemia and microscopic haematuria were frequently noted.

Complications occurred in $63 \%$ of patients, with emboli being the most common. The sur- 
gery rate was $23.5 \%$. The overall mortality rate was $18 \%$. Of the cases, $76.5 \%$ were cured with antibiotics alone, including a third-generation cephalosporin, the current recommended therapy, or a combination of a penicillin and an aminoglycoside. Antibiotic therapy duration of at least 4 weeks is recommended. Surgical therapy is usually required for haemodynamic reasons.

The presence of A.actinomycetemcomitans on aspiration of the thigh abscess raised the possibility of endocarditis, though no murmur was clinically detectable and blood cultures were negative. This case is a most unusual initial presentation of A.actinomycetemcomitans endocarditis.

\section{References}

1. Pasturel L, Casalta J, Habib G, et al. Actinobacillus actinomycetemcomitans endocarditis. Clin Microbiol Infect 2004;10:
98-118.

2. Kaplan A, Weber D, Oddone E, Perfect J. Infection due to Actinobacillus actinomycetemcomitans: 15 cases and review. Rev Infect Dis 1989;1:46-63.

3. Burgher L, Loomis G, Ware F. Systemic infection due to Actinobacillus actinomycetemcomitans. Am J Clin Pathol 1973; 60:412-5.

4. Mitchell RG, Gillepsie WA. Bacterial endocarditis due to an actinobacillus. J Clin Pathol 1964;17:511-2. 